\title{
Development and validation of nomogram to predict severe illness requiring intensive care follow up in hospitalized COVID-19 cases
}

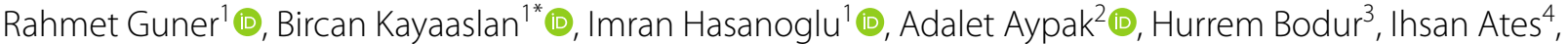

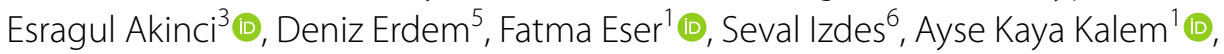 \\ Aliye Bastug ${ }^{3}$, Aysegul Karalezli ${ }^{7}$, Aziz Ahmet Surel ${ }^{8}$, Muge Ayhan ${ }^{2}{ }^{\circledR}$, Selma Karaahmetoglu ${ }^{4}$,

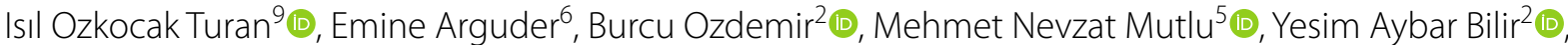

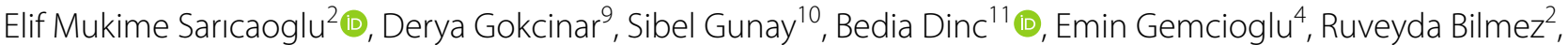 \\ Omer Aydos², Dilek Asilturk², Osman Inan ${ }^{4}$ and Turan Buzgan ${ }^{1}$ (D)
}

\begin{abstract}
Background: Early identification of severe COVID-19 patients who will need intensive care unit (ICU) follow-up and providing rapid, aggressive supportive care may reduce mortality and provide optimal use of medical resources. We aimed to develop and validate a nomogram to predict severe COVID-19 cases that would need ICU follow-up based on available and accessible patient values.

Methods: Patients hospitalized with laboratory-confirmed COVID-19 between March 15, 2020, and June 15, 2020, were enrolled in this retrospective study with 35 variables obtained upon admission considered. Univariate and multivariable logistic regression models were constructed to select potential predictive parameters using 1000 bootstrap samples. Afterward, a nomogram was developed with 5 variables selected from multivariable analysis. The nomogram model was evaluated by Area Under the Curve (AUC) and bias-corrected Harrell's C-index with 95\% confidence interval, Hosmer-Lemeshow Goodness-of-fit test, and calibration curve analysis.
\end{abstract}

Results: Out of a total of 1022 patients, 686 cases without missing data were used to construct the nomogram. Of the 686, 104 needed ICU follow-up. The final model includes oxygen saturation, CRP, PCT, LDH, troponin as independent factors for the prediction of need for ICU admission. The model has good predictive power with an AUC of 0.93 (0.902-0.950) and a bias-corrected Harrell's C-index of 0.91 (0.899-0.947). Hosmer-Lemeshow test p-value was 0.826 and the model is well-calibrated $(p=0.1703)$.

Conclusion: We developed a simple, accessible, easy-to-use nomogram with good distinctive power for severe illness requiring ICU follow-up. Clinicians can easily predict the course of COVID-19 and decide the procedure and facility of further follow-up by using clinical and laboratory values of patients available upon admission.

Keywords: COVID-19, Nomogram, Severity Score, Predictive Factors, Intensive Care

*Correspondence: drbican@gmail.com

${ }^{1}$ Department of Infectious Disease and Clinical Microbiology, Ankara

Yildirim Beyazit University, Ankara City Hospital, Bilkent Street no:1,

Ankara 06800, Turkey

Full list of author information is available at the end of the article

\section{Background}

The world has been under threat of the novel coronavirus disease (COVID-19) since the last days of 2019. Although the disease has a wide clinical spectrum from asymptomatic infection to critically ill [1], a small number of

(c) The Author(s) 2021. Open Access This article is licensed under a Creative Commons Attribution 4.0 International License, which permits use, sharing, adaptation, distribution and reproduction in any medium or format, as long as you give appropriate credit to the original author(s) and the source, provide a link to the Creative Commons licence, and indicate if changes were made. The images or other third party material in this article are included in the article's Creative Commons licence, unless indicated otherwise in a credit line to the material. If material is not included in the article's Creative Commons licence and your intended use is not permitted by statutory regulation or exceeds the permitted use, you will need to obtain permission directly from the copyright holder. To view a copy of this licence, visit http://creativecommons.org/licenses/by/4.0/. The Creative Commons Public Domain Dedication waiver (http://creativeco mmons.org/publicdomain/zero/1.0/) applies to the data made available in this article, unless otherwise stated in a credit line to the data. 
COVID-19 patients experience a severe illness that can result in death. The Chinese Center for Disease Control and Prevention reported mild disease, serious illness, and critical illness as $81 \%, 14 \%$, and $5 \%$ in 44.672 confirmed cases, respectively [2]. The case fatality rate was reported as $2.3 \%$ but had increased to $49.0 \%$ in critical cases.

Since there is no specific treatment for the new coronavirus (severe acute respiratory syndrome coronavirus-2 [SARS-CoV-2]) so far, the early recognition of patients who will worsen and the provision of aggressive supportive treatment is the essential point of patient management. Therefore, early detection of patients whose illness will progress helps the physician to decide whether the patient should be followed up in the hospital or outpatient clinic or if there is a need for transferring to a referral center. Additionally, early detection of the disease severity with a predictive calculation tool can optimize the duration of hospitalization, especially in countries with limited resources in terms of hospital beds and finances. Early identification of patients with a simple and easy-to-use method will save time for the physicians and patients for providing rapid supportive care and reduce the mortality rate. Patients who are predicted not to need an intensive care unit (ICU) can be discharged from the hospital earlier. Herein, we aimed to construct and validate a nomogram and a web-based calculation tool that incorporated demographic, clinical characteristics, and initial laboratory results at admission to hospital for predicting the development of severe illness that will require ICU follow up.

\section{Methods}

\section{Study design and participants}

This retrospective cohort study was carried out in Ankara City Hospital, set apart as the main pandemic response center in Ankara with 3810 beds, of which 696 are intensive care beds. The ethical approval was obtained from Ankara City Hospital Ethical Committee 1 . Verbal consent was obtained after the patients were informed that their medical records would only be used in scientific studies after anonymization of their personal information. All patients older than 18 years who were hospitalized with the diagnosis of COVID-19 infection between March 15, 2020, and June 15, 2020, were included in the study. Only COVID-19 patients with the definite diagnosis were included in the study. The diagnosis was confirmed with polymerase chain reaction (PCR) for SARS-CoV-2 performed based on the protocol established by the World Health Organization (WHO) interim guideline [3]. Patients were monitored up to June 30, 2020, the final date of follow up. Patients with a negative SARS-CoV-2 test even if typical chest computed tomography $(\mathrm{CT})$ findings and those who were still in hospital at the moment of final date of follow up (if no death or discharge) were excluded.

The patients with a severe and critical illness were candidate to ICU follow-up based on the WHO COVID-19 disease severity classification. Patients with pneumonia and one of the following: $>30$ breaths/ min; severe respiratory distress; or $\mathrm{O}_{2}$ saturation $\left(\mathrm{SpO}_{2}\right)<90 \%$ on room air were considered severe. Patients were considered critical if they had acute respiratory distress syndrome (ARDS) or other respiratory failure requiring mechanical ventilation, or septic shock, and/or organ failure requiring ICU follow up [1]. The decision of ICU admission was made by intensive care specialists. ICU admission criteria were respiratory rate $\geq 30, \mathrm{SpO}_{2}<90 \%$ or partial oxygen pressure $\left(\mathrm{PaO}_{2}\right)<70 \mathrm{mmHg}$ on room air despite nasal oxygen support of $5 \mathrm{lt} / \mathrm{min}$ or above, $\mathrm{PaO}_{2} /$ fraction of inspired oxygen $\left(\mathrm{FiO}_{2}\right)<300$. The primary outcome was defined as severe illness that required ICU follow up. The patients were classified as cases who required ICU follow up and those who did not require ICU follow up based on disease severity.

\section{Collecting and processing data}

To collect data, a special form was created for COVID-19 patients, containing information of patients at the admission and follow up. The parameters included in special patient forms were age, gender, smoking status, comorbid diseases, the symptoms of fever and dyspnea, oxygen saturation $\left(\mathrm{SpO}_{2}\right)$, quick sequential organ failure assessment (qSOFA) at admission. The forms also included following laboratory and radiological tests: complete blood counts, serum biochemistry, C-reactive protein (CRP), procalcitonin (PCT), coagulation tests, ferritin, D-dimer, troponin I, and chest CT. The clinical outcomes were defined as requirement of ICU or discharge from hospital.

Data were collected prospectively. In case of patient death or discharge, all the missing laboratory records in patient files were completed from the hospital database and registered in an electronic recording system and uploaded collaboratively to an online database created specifically for COVID-19 patients. Data cut-off for the study was June 30, 2020. Data were recorded to the system by the physicians who followed up the patients from different departments including infectious disease, internal medicine, respiratory disease, and anesthesiology and reanimation. After patient records were compiled, the data was checked by two independent controllers who were infectious disease physicians. Patients with more than $30 \%$ missing data were not included in the study. 


\section{Potential predictive parameters and outcome}

For the development of a functional nomogram, patient data obtained on the day of hospitalization were used. The predictors were selected from the factors that affect the prognosis of the patients such as age and the presence of comorbidities, and clinical features and easily accessible, practical, and quickly performed laboratory parameters. The potential predictive parameters were determined as age, gender, the presence of fever and dyspnea, and qSOFA on admission, clinical risk factors (comorbidities including hypertension, coronary arterial disease, diabetes mellitus, chronic lung disease, malignity, and number of comorbidities), $\mathrm{SpO} 2$ and laboratory parameters which are found significant covariates on COVID-19 infection including white blood cell (WBC), monocyte, neutrophil to lymphocyte ratio (NLR), hemoglobin (HGB), platelet count, urea, creatinine, glomerular filtration rate (GFR), albumin, aspartate transaminase (AST), alanine transaminase (ALT), lactate dehydrogenase (LDH), creatine kinase (CK), troponin I, CRP, PCT, ferritin, prothrombin time (PT), activated partial thromboplastin time (aPTT), D-dimer, international normalized ratio (INR) and fibrinogen, and presence of bilateral infiltration on chest CT. A total of 35 predictors were included in the construction of nomogram in the beginning. After determining potential predictors, their association with ICU hospitalization was investigated.

\section{Statistical analysis}

Statistical analyses were performed using $\mathrm{R}$ software version 4.0.4 ( $\mathrm{R}$ Foundation for Statistical Computing, Vienna, Austria) and IBM SPSS Statistics version 23.0 for Windows (IBM Corporation, Armonk, NY). Missing data pattern and mechanism were evaluated using $\mathrm{R}$ packages VIM, mice, MissMech, and BaylorEdPsych [4-7]. Listwise deletion (complete-case method) was applied for handling missing data due to the MNAR mechanisms in numerical measurements. Laboratory parameters were discretized using Receiver Operating Characteristic (ROC) analysis. Thereafter, Youden's J Index was used for determining optimal cut-off points of the numerical variables in predicting ICU admission. Descriptive statistics were presented as median with interquartile range (IQR) for continuous variables since the distribution of the variables were skewed and contains extreme values. Frequency and percentages were presented as descriptive statistics for categorical variables. Mann-Whitney U test was used in the comparison of continuous variables between patients admitted to ICU and those without need for ICU follow up due to the violation of the parametric test assumptions. Pearson $\chi^{2}$ test was used for testing independence between ICU admission status and other categorical variables when test requirements were satisfied. Otherwise, Fisher's Exact test was used.

To estimate ICU admission status, univariate logistic models were constructed using bootstrap sampling with 1000 samples and bootstrap estimated $\mathrm{p}$ values were evaluated. The variables with bootstrapped $\mathrm{p}$-value below 0.25 as considered candidate variable for multivariable analysis [8]. Numeric variables which were included in the multivariable analysis are evaluated for linearity in logit and multicollinearity was investigated using Variance inflation factor (VIF) before applying the variable selection method [9]. Both univariate and multivariable logistic regression analysis were carried out using $\mathrm{R} r \mathrm{rms}$ package [10]. Fast backward elimination method for variable selection was carried out with bootstrap sampling (1000 successful bootstrap samples) to develop a parsimonious model for predicting ICU admission. Estimations obtained from multivariable model was based on penalized maximum likelihood estimations with best penalty parameter obtained using pentrace function in $\mathrm{R}$ rms package. In addition, final model is selected according to the Akaike Information Criteria (AIC).

Selected variables were represented as odds ratio (OR) with bootstrapped 95\% confidence interval (CI) and two-tailed $p$-values. Discrimination was evaluated using bias-corrected Harrell's Concordance index (C-index). Bias-corrected Harrel's C-index was calculated from rms package validate function with 1000 successful bootstrap samples. Validated model is checked for multicollinearity. Hence, VIF values of all the predictor variables in the multivariable model were below 5 . In addition, linearity in logit assumption was satisfied. Nomogram was constructed based on the final validated model for estimating the admission to ICU and provided a quantitative tool for physicians to assess the individual probability of ICU admission. In addition, model's discriminative power was evaluated with ROC analysis using R pROC package [11]. The Area Under the Curve (AUC) was obtain with 95\% Hereafter in the article, "corrected C-index" will be used to indicate that AUC value/C-index was obtained from bootstrap samples and bias-corrected, and "AUC" will be used to indicate crude AUC value/C-index which was obtained from one ROC Curve.

Calibration plots were developed to assess the predictive accuracy and agreement between predicted and observed ICU admission with 1000 bootstrap samples and calibration curve analyses were performed in addition to Hosmer-Lemeshow goodness of fit evaluation. In addition, both the unreliability test and the calibration test are performed to evaluate good calibration.

In addition, false-negative (i.e. not admitting the patient in the ICU when the patient needs intensive care) is far more harmful than the false positive (i.e. admitting 


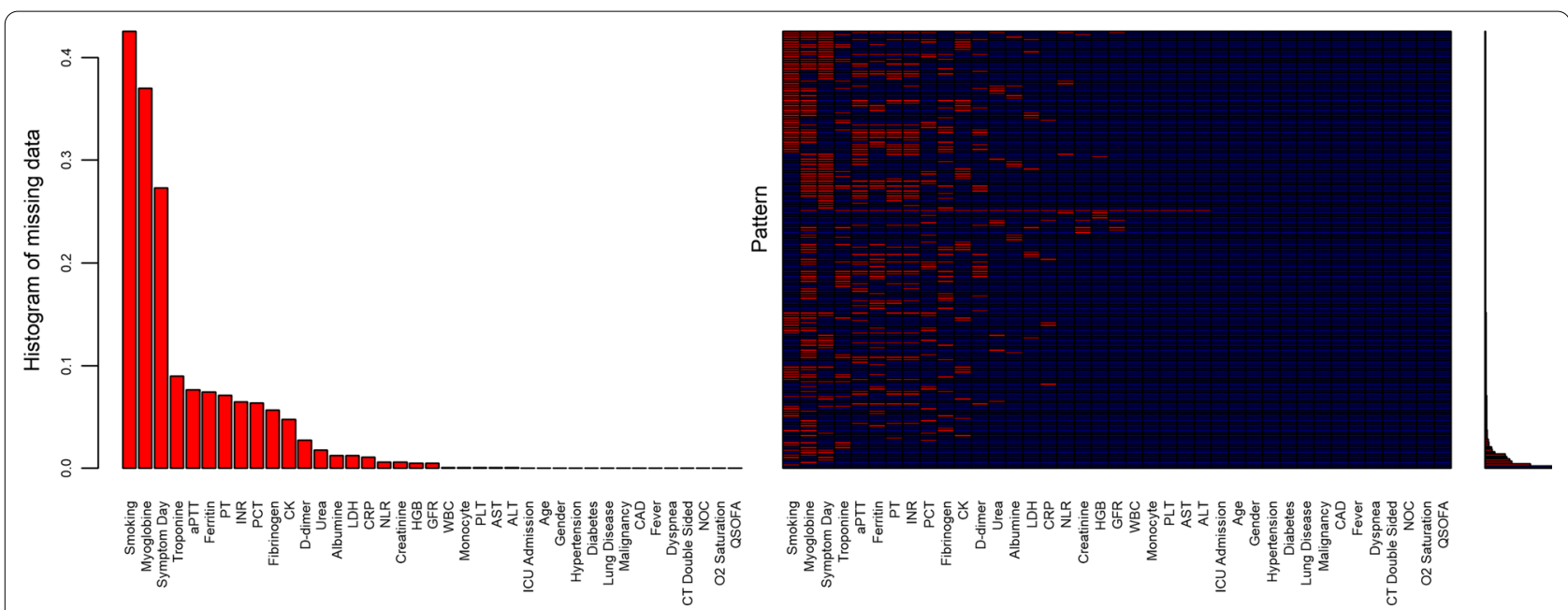

Fig. 1 Evaluation of missing data mechanism. The first plot represents the distribution of the missing data proportions in the variables considered in the study. The second plot is a heatmap represent the missing data pattern in the study. The third plot is the vertical version of the first plot which represents the missing data proportions in the heatmap

the patient in the ICU when the patient doesn't need intensive care at all) in the present study. Therefore, decision curve analysis was performed since the possibility of ICU need of the patient is more crucial for patients' wellbeing. In a decision curve analysis, a low-risk threshold probability might indicate that delaying the ICU admission is far more harmful than early admission; a higher threshold might indicate that waiting the parameters to reach critical levels is relatively more harmful than unnecessary ICU admission.

All analysis related to the evaluating classification performance and calibration of the prognostic accuracy of the nomogram model were performed according to TRIPOD guidelines [12].

\section{Results}

\section{Characteristics of study cohort}

Study included 1022 patients with laboratory confirmed COVID-19. The dataset consists of these 1022 patient records contained missing data ranged between $0.1 \%$ and $42 \%$. Variables which consist of more than $20 \%$ percent of missing data were excluded from the analysis. The proportion of missing data ranged from $0.1 \%$ to $9 \%$ after exclusion of the variables which consist of more than $\% 20$ missing observations (smoking status, myoglobin, symptom duration before hospital admission). The MCAR test in the R MissMech package [4-7] was used to assess whether the missing data mechanism is Missing Completely at Random (MCAR). MCAR hypothesis was rejected at 0.05 level. Therefore, after list-wise deletion 686 cases out of 1022 patient remained for further analysis. Brief overview of the missing data structure is represented graphically (Fig. 1).

The demographic characteristics, comorbidities, and initial laboratory parameters of patients are shown in Table 1. Of the 686 patients, $104(15.2 \%)$ required ICU follow up during hospitalization. There was no difference in gender between the groups $(p=0.057), 52.4 \%$ of patients who did not need ICU follow up and $62.5 \%$ of those who needed ICU follow up were male. The median age was higher in the patients requiring ICU follow up (67, IQR 30-54) than those who did not need ICU follow up (42, IQR 54-76) $(\mathrm{p}<0.001)$. The patients who required ICU follow up had significantly higher rates of hypertension $(40.4 \%$ vs $12.8 \%)$, coronary arterial disease $(20.2 \%$ vs $6.4 \%)$, diabetes mellitus ( $29.3 \%$ vs $10.1 \%)$, chronic pulmonary disease $(21.2 \%$ vs $6.4 \%)$, and malignity (9.6\% vs $1.9 \%)$ compared to those who did not need ICU admission (for all, $\mathrm{p}<0.001)$. Fever $(48.1 \%$ vs $34.0 \%, \mathrm{p}=0.006$ ) and dyspnea (59.9\% vs $19.4 \%, \mathrm{p}<0.001)$ were significantly more frequent on admission in the patients who needed ICU follow up. Laboratory features of two groups were compared. All initial parameters except ALT and aPTT were significantly different between the two groups (for all parameters, $\mathrm{p}<0.001$ ).

Univariate analysis indicates that common laboratory features have possible effect on the patient's requirement of intensive care as well as the patient characteristics such as age, gender and comorbidities (Table 2).

\section{Potential predictive factors for ICU admission}

A total of 35 predictor were chosen for the development of nomogram predicting of ICU admission in 
Table 1 Demographic, clinical characteristics and laboratory parameters of COVID-19 patients

\begin{tabular}{|c|c|c|c|}
\hline & $\begin{array}{l}\text { Patient did not need ICU follow up } \\
(\mathrm{N}=582)\end{array}$ & Patient need ICU follow up $(\mathrm{N}=104)$ & $p$ \\
\hline Age. median (IQR), years & $42(30-54)$ & $67(54-76)$ & $<0.001$ \\
\hline Age, years & & & $<0.001$ \\
\hline$\geq 56.5$ & $121(20.8)$ & $76(73.1)$ & \\
\hline$<56.5$ & $461(79.2)$ & $28(26.9)$ & \\
\hline Gender, female sex & $277(47.6)$ & $39(37.5)$ & 0.057 \\
\hline Hypertension & $106(12.8)$ & $42(40.4)$ & $<0.001$ \\
\hline Diabetes & $58(10.1)$ & $31(29.3)$ & $<0.001$ \\
\hline Chronic pulmonary disease & $37(6.4)$ & $22(21.2)$ & $<0.001$ \\
\hline Malignity & $11(1.9)$ & $10(9.6)$ & $<0.001$ \\
\hline Coronary arterial disease & $37(6.4)$ & $21(20.2)$ & $<0.001$ \\
\hline Fever on admission & $198(34.0)$ & $50(48.1)$ & 0.006 \\
\hline Dyspnea on admission & $113(19.4)$ & $62(59.9)$ & \\
\hline Oxygen saturation, \% & & & $<0.001$ \\
\hline$>94.5$ & $463(79.5)$ & $22(21.2)$ & \\
\hline$\leq 94.5$ & $119(20.4)$ & $82(78.8)$ & \\
\hline qSOFA & & & $<0.001$ \\
\hline 0 & $553(95.0)$ & $38(36.5)$ & \\
\hline$\geq 1$ & $29(5.0)$ & $66(63.5)$ & \\
\hline White blood cell, $\times 10^{9} / \mathrm{L}$ & & & 0.019 \\
\hline$\geq 7.4$ & $85(14.6)$ & $36(34.6)$ & \\
\hline$<7.4$ & $497(85.4)$ & $68(65.5)$ & \\
\hline Monocyte, $\times 10^{9} / \mathrm{L}$ & & & 0.006 \\
\hline$>0.23$ & $506(86.9)$ & $70(67.5)$ & \\
\hline$\leq 0.23$ & $76(13.1)$ & $34(32.7)$ & \\
\hline NLR & & & $<0.001$ \\
\hline$\geq 3.5$ & $173(29.7)$ & $77(74.0)$ & \\
\hline$<3.5$ & $409(70.3)$ & $27(26.0)$ & \\
\hline Hemoglobin, g/L & & & $<0.001$ \\
\hline$>12.5$ & $458(78.7)$ & $61(58.7)$ & \\
\hline$\leq 12.5 \mathrm{~g} / \mathrm{L}$ & $124(21.3)$ & $43(41.3)$ & \\
\hline Platelet count, $\times 10^{9} / \mathrm{L}$ & & & 0.031 \\
\hline$>155$ & $492(84.5)$ & $73(70.2)$ & \\
\hline$\leq 155$ & $90(15.5)$ & $31(29.9)$ & \\
\hline $\mathrm{GFR}, \mathrm{ml} / \mathrm{min} / 1.73 \mathrm{~m} 2$ & & & $<0.001$ \\
\hline$>96.2$ & $369(63.4)$ & $24(23.1)$ & \\
\hline$\leq 96.2$ & $213(36.6)$ & $80(76.9)$ & \\
\hline Aspartate transaminase, $\mathrm{U} / \mathrm{L}$ & & & $<0.001$ \\
\hline$\geq 28.5$ & $166(22.5)$ & $79(76.0)$ & \\
\hline$<28.5$ & $416(71.5)$ & $25(24.0)$ & \\
\hline Alanine transaminase, $\mathrm{U} / \mathrm{L}$ & & & 0.164 \\
\hline$\geq 40.5$ & $128(22.0)$ & $33(317)$ & \\
\hline$<40.5$ & $454(78.0)$ & $71(68.3)$ & \\
\hline Albumin, $\mathrm{g} / \mathrm{L}$ & & & $<0.001$ \\
\hline$>42.5$ & $417(71.5)$ & $23(22.1)$ & \\
\hline$\leq 42.5$ & $166(28.5)$ & $81(77.9)$ & \\
\hline Creatine kinase, $U / L$ & & & 0.002 \\
\hline$\geq 141.5$ & $136(23.4)$ & $45(43.3)$ & \\
\hline$<141.5$ & $446(76.6)$ & $59(56.7)$ & \\
\hline Lactate dehydrogenase, U/L & & & $<0.001$ \\
\hline
\end{tabular}


Table 1 (continued)

\begin{tabular}{|c|c|c|c|}
\hline & $\begin{array}{l}\text { Patient did not need ICU follow up } \\
(\mathrm{N}=582)\end{array}$ & Patient need ICU follow up $(\mathrm{N}=104)$ & $p$ \\
\hline$\geq 286.5$ & $94(16.2)$ & $71(68.3)$ & \\
\hline$<286.5$ & $488(83.8)$ & $33(31.7)$ & \\
\hline C-reactive protein, $\mathrm{g} / \mathrm{L}$ & & & $<0.001$ \\
\hline$\geq 0.0275$ & $123(21.1)$ & $81(77.9)$ & \\
\hline$<0.0275$ & $459(78.9)$ & $23(22.1)$ & \\
\hline Procalcitonin, pg/mL & & & $<0.001$ \\
\hline$\geq 0.085$ & $71(12.2)$ & $71(68.7)$ & \\
\hline$<0.085$ & $511(87.8)$ & $33(31.7)$ & \\
\hline Troponin, ng/L & & & $<0.001$ \\
\hline$\geq 5.9$ & $113(19.4)$ & $78(75.0)$ & \\
\hline$<5.9$ & $469(80.6)$ & $26(25.0)$ & \\
\hline Prothrombin time, sec & & & $<0.001$ \\
\hline$\geq 12.5$ & $153(26.3)$ & $63(60.6)$ & \\
\hline$<12.5$ & $429(73.7)$ & $41(39.4)$ & \\
\hline aPTT, sec & & & 0.499 \\
\hline$\geq 26.2$ & $162(27.8)$ & $40(38.5)$ & \\
\hline$<26.2$ & $72.8(72.2)$ & $64(61.5)$ & \\
\hline INR & & & $<0.001$ \\
\hline$\geq 1.06$ & $183(31.4)$ & $68(65.4)$ & \\
\hline$<1.06$ & $399(68.6)$ & $36(34.6)$ & \\
\hline Ferritin, $\mu \mathrm{g} / \mathrm{L}$ & & & $<0.001$ \\
\hline$\geq 227.5$ & $155(26.6)$ & $70(673)$ & \\
\hline$<227.5$ & $427(73.4)$ & $34(32.7)$ & \\
\hline D-dimer, mg/L & & & $<0.001$ \\
\hline$\geq 0.535$ & $210(36.1)$ & 88 (84.6) & \\
\hline$<0.535$ & $372(63.9)$ & $16(15.4)$ & \\
\hline Fibrinogen, g/L & & & $<0.001$ \\
\hline$\geq 3.40$ & $210(36.1)$ & $76(73.1)$ & \\
\hline$<3.40$ & $372(63.9)$ & $28(26.9)$ & \\
\hline Urea, mg/dL & & & $<0.001$ \\
\hline$\geq 36.1$ & $116(19.9)$ & $56(53.8)$ & \\
\hline$<36.1$ & $466(80.1)$ & $48(46.2)$ & \\
\hline Creatinine, mg/dL & & & $<0.001$ \\
\hline$\geq 0.96$ & $125(21.5)$ & $41(39.4)$ & \\
\hline$<0.96$ & $457(78.5)$ & $63(60.6)$ & \\
\hline Bilateral infiltration on CT & $250(43.0)$ & 79 (70.6) & $<0.001$ \\
\hline
\end{tabular}

IQR, Interquartile range; NLR, Neutrophil to lymphocyte ratio; aPTT, Activated partial thromboplastin time; INR, International normalized ratio; $\mathrm{CT}$, Computed tomography; GFR, Glomerular filtration rate; qSOFA, quick sequential organ failure assessment

hospitalized patients with COVID-19. All predictors have p-value below 0.25 . Therefore, they were all considered as candidate variables for multivariable analysis except QSOFA score due to sparsity and quasi-complete separation problem (All the patients whose QSOFA score equals 2 had been admitted to the $\mathrm{ICU})$.

\section{Construction of nomogram predicting ICU admission status}

The nomogram was constructed using the data obtained from 686 patients' records. Afterwards, all laboratory parameters were discretized by using optimal cut-off points obtained from ROC analysis with Youden's J Index. To construct multivariable nomogram model for estimating ICU admission status of inpatients, first candidate variables were selected using univariate analysis 
Table 2 Univariate analysis of potential predictive parameters for ICU admission in hospitalized COVID-19 patients

\begin{tabular}{|c|c|c|c|c|c|}
\hline & Beta & OR & $95 \% \mathrm{CI} \mathrm{L}$ & $95 \% \mathrm{CIU}$ & $p$ \\
\hline Age, 56.5 years and older & 2.34 & 10.34 & 6.42 & 17.78 & $<0.0001$ \\
\hline Gender. female sex & -0.42 & 0.66 & 0.43 & 1.03 & 0.0577 \\
\hline Hypertension & 1.11 & 3.04 & 1.96 & 4.91 & $<0.0001$ \\
\hline Diabetes & 1.35 & 3.84 & 2.30 & 6.27 & $<0.0001$ \\
\hline Chronic pulmonary disease & 1.37 & 3.95 & 2.01 & 6.98 & $<0.0001$ \\
\hline Malignity & 1.71 & 5.52 & 2.04 & 13.65 & 0.0004 \\
\hline Coronary arterial disease & 1.32 & 3.73 & 2.00 & 6.42 & $<0.0001$ \\
\hline Number of comorbidities & 0.81 & 2.24 & 1.63 & 3.05 & $<0.0001$ \\
\hline Fever on admission & 0.59 & 1.80 & 1.14 & 2.75 & 0.0098 \\
\hline Dyspnea on admission & 1.81 & 6.13 & 3.87 & 9.65 & $<0.0001$ \\
\hline Oxygen saturation, $94.5 \%$ and below & 2.67 & 14.50 & 8.93 & 25.80 & $<0.0001$ \\
\hline White blood cell, $7.4 \times 10^{9} / \mathrm{L}$ and above & 1.13 & 3.10 & 1.83 & 5.00 & $<0.0001$ \\
\hline Monocyte, $0.23 \times 10^{9} / \mathrm{L}$ and below & 1.17 & 3.23 & 1.95 & 5.28 & $<0.0001$ \\
\hline NLR, 3.5 and above & 1.91 & 6.74 & 4.25 & 11.37 & $<0.0001$ \\
\hline Hemoglobin, $12.5 \mathrm{~g} / \mathrm{L}$ and below & 0.96 & 2.60 & 1.69 & 4.02 & $<0.0001$ \\
\hline Platelet count, $155 \times 10^{9} / \mathrm{L}$ and below & 0.84 & 2.32 & 1.41 & 3.67 & 0.0006 \\
\hline Urea, $36.1 \mathrm{mg} / \mathrm{dL}$ and above & 1.55 & 4.69 & 3.06 & 7.42 & $<0.0001$ \\
\hline Creatinine, $0.96 \mathrm{mg} / \mathrm{dL}$ and above & 0.87 & 2.38 & 1.54 & 3.69 & $<0.0001$ \\
\hline GFR, $96.2 \mathrm{ml} / \mathrm{min} / 1.73 \mathrm{~m} 2$ and below & 1.75 & 5.78 & 3.47 & 9.68 & $<0.0001$ \\
\hline Aspartate transaminase, $28.5 \mathrm{U} / \mathrm{L}$ and above & 2.07 & 7.92 & 5.02 & 13.39 & $<0.0001$ \\
\hline Alanine transaminase, $40.5 \mathrm{U} / \mathrm{L}$ and above & 0.50 & 1.65 & 1.02 & 2.64 & 0.0369 \\
\hline Albumin, $42.5 \mathrm{~g} / \mathrm{L}$ and below & 2.18 & 8.83 & 5.66 & 15.52 & $<0.0001$ \\
\hline Creatine kinase, $141.5 \mathrm{U} / \mathrm{L}$ and above & 0.92 & 2.50 & 1.61 & 3.85 & $<0.0001$ \\
\hline Lactate dehydrogenase, $286.5 \mathrm{U} / \mathrm{L}$ and above & 2.41 & 11.17 & 6.94 & 18.72 & $<0.0001$ \\
\hline C-reactive protein, $0.0275 \mathrm{~g} / \mathrm{L}$ and above & 2.58 & 13.14 & 8.42 & 22.96 & $<0.0001$ \\
\hline Procalcitonin, $0.085 \mathrm{pg} / \mathrm{mL}$ and above & 2.74 & 15.49 & 10.19 & 25.67 & $<0.0001$ \\
\hline Troponin, $5.9 \mathrm{ng} / \mathrm{L}$ and above & 2.52 & 12.45 & 7.90 & 22.09 & $<0.0001$ \\
\hline Prothrombin time, $12.5 \mathrm{~s}$ and above & 1.46 & 4.31 & 2.86 & 6.52 & $<0.0001$ \\
\hline aPTT, 26.2 $\mathrm{s}$ and above & 0.48 & 1.62 & 1.05 & 2.43 & 0.0230 \\
\hline INR, 1.06 and above & 1.42 & 4.12 & 2.67 & 6.54 & $<0.0001$ \\
\hline Ferritin, $227.5 \mu \mathrm{g} / \mathrm{L}$ and above & 1.74 & 5.67 & 3.73 & 9.13 & $<0.0001$ \\
\hline D-dimer, $0.535 \mathrm{mg} / \mathrm{L}$ and above & 2.28 & 9.74 & 5.95 & 18.92 & $<0.0001$ \\
\hline Fibrinogen, $3.40 \mathrm{~g} / \mathrm{L}$ and above & 1.57 & 4.81 & 3.10 & 7.94 & $<0.0001$ \\
\hline Bilateral infiltration on CT & 1.43 & 4.20 & 2.68 & 7.33 & $<0.0001$ \\
\hline qSOFA:1 & 2.67 & 14.49 & 9.38 & 22.95 & $<0.0001$ \\
\hline qSOFA:2 & 3.26 & 25.92 & 15.96 & 36.14 & $<0.0001$ \\
\hline
\end{tabular}

NLR, Neutrophil to lymphocyte ratio; aPTT, Activated partial thromboplastin time; INR, International normalized ratio; CT, Computed tomography; GFR, Glomerular filtration rate; qSOFA, quick sequential organ failure assessment

(Table 2). All variables had p value below 0.25 and considered for the multivariable analysis. After candidate variables were chosen, multivariable model was constructed with 34 variables including demographical, clinical and laboratory parameters. The parameters included in the initial model were age, gender, hypertension, coronary arterial disease, diabetes mellitus, chronic pulmonary disease, malignity, number of comorbidities, fever and dyspnea on admission, $\mathrm{SpO} 2, \mathrm{WBC}$, monocyte, NLR, HGB, platelet count, urea, creatinine, GFR, AST, ALT, albumin, LDH, CK, CRP, PCT, ferritin, troponin, PT, aPTT, INR, D-dimer, fibrinogen and bilateral infiltration on CT. Final model was selected according to the Akaike Information Criteria (AIC), and only SpO2, LDH, CRP, PCT and troponin, which were shown to be independent risk factors for predicting ICU admission, were included. The present nomogram calculates the risk for requirement of ICU in hospital admission of patients using these 5 parameters (Fig. 2). Additional information on score assignment for each variable and calculation the risk for 


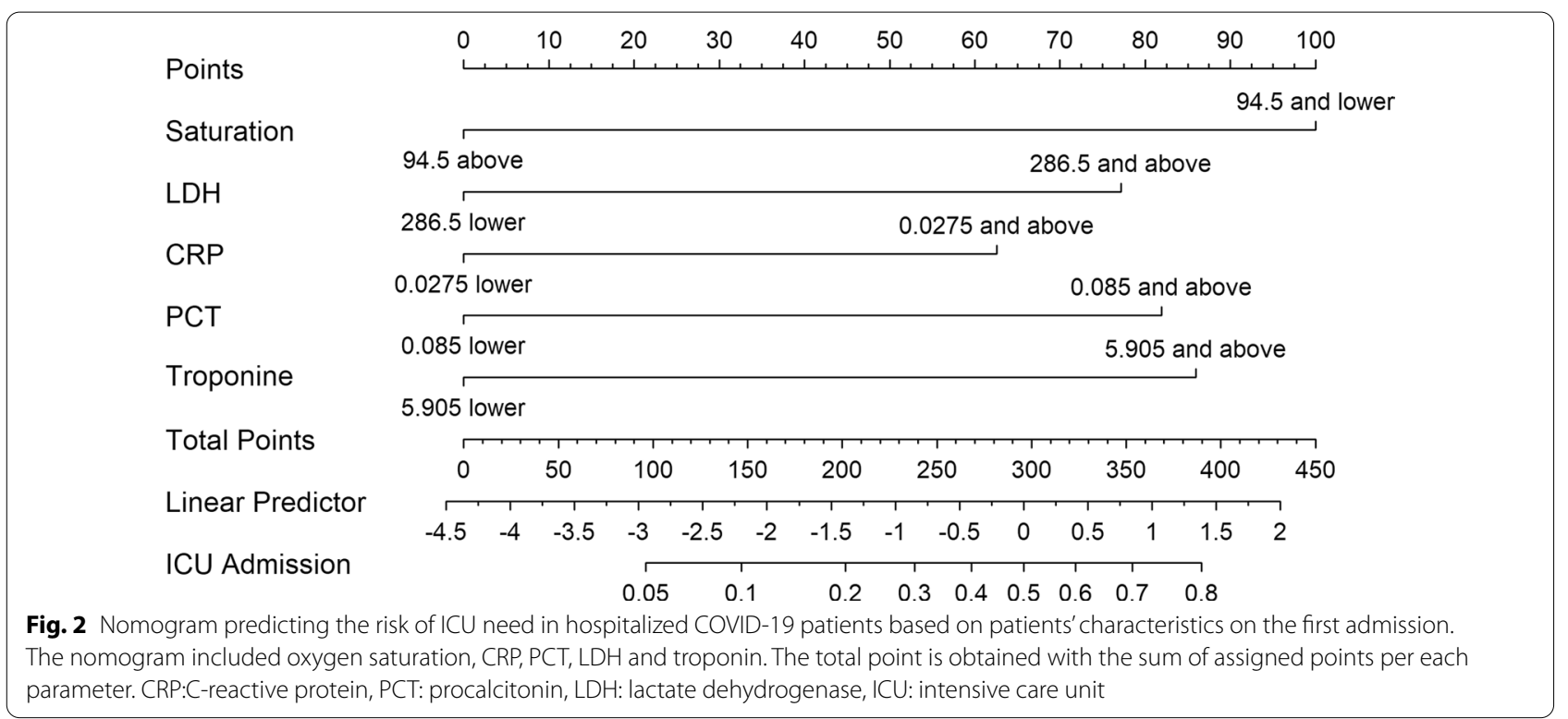

ICU admission based on total point is shown in Additional file 1 . According to the nomogram model, the risk of ICU admission is 4.4 (95\% CI 2.48-7.72) times higher in patients with oxygen saturation equal and below $94.5 \%$ compared to the patients with oxygen saturation above 94.5\% $(\mathrm{p}<0.0001)$. In addition, the risk is $3.1(95 \% \mathrm{CI}$ 1.76-5.53) times higher in patients with LDH level equal and above $286.5 \mathrm{U} / \mathrm{L}$ compared to the patients with $\mathrm{LDH}$ level below $286.5 \mathrm{U} / \mathrm{L}(\mathrm{p}<0.0001)$ while it is 2.5 (95\% CI 1.37-4.63) times higher in patients with CRP level equal and above $0.0275 \mathrm{~g} / \mathrm{L}$ compared to the patients with CRP level below $0.0275 \mathrm{~g} / \mathrm{L}(\mathrm{p}=0.0029)$. Whereas the risk is 3.4 (95\% CI 1.89-5.94) times higher in patients with PCT level equal and above $0.085 \mathrm{pg} / \mathrm{mL}$ compared to the patients with PCT level below $0.085 \mathrm{pg} / \mathrm{mL}(\mathrm{p}<0.0001)$, it is 3.6 (95\% CI 2.03-6.22) times higher in patients with troponin level equal and above $5.9 \mathrm{ng} / \mathrm{L}$ compared to the patients with troponin level below $5.9 \mathrm{ng} / \mathrm{L}(\mathrm{p}<0.0001)$ (Table 3).

Based on these 5 independent risk factors included in the nomogram for ICU admission, a web-based calculation tool was constructed. The clinician can easily access the calculation tool using the online website at https://achcovid19.com/prj/f?p=126:1.

\section{The accuracy of nomogram prediction model validation}

The nomogram model had a significantly high predictive value for the development of ICU needs in hospitalized patients. The model had an AUC of 0.93 (0.902-0.950) (Fig. 3a). In addition, we evaluate the validation of the final model using bootstrap resampling method and obtained corrected C-index of the nomogram as 0.91 (95\% CI 0.899-0.947) which implies exceptionally good discriminative value for differentiating inpatients who needed ICU follow up from those who did not.

Furthermore, the decision curve revealed that when threshold probability is between 0.15 and 0.85 , predicting ICU admission by using our nomogram model would provide higher net benefit than the admitting all the patients to the ICU (All) or admitting none of the patients to the ICU (None) (Fig. 4).

Table 3 Estimation of the Odds ratios with 95\% confidence intervals of the multivariable nomogram model

\begin{tabular}{|c|c|c|c|c|}
\hline Variable & Odds ratio & $\% 95 \mathrm{Cl}$ lower bound & $\% 95 \mathrm{Cl}$ upper bound & $\mathrm{p}$ \\
\hline Intercept & 0.013 & 0.0069 & 0.0236 & $<0.0001$ \\
\hline Oxygen saturation (SpO2), 94.5\% and below & 4.372 & 2.4769 & 7.7168 & $<0.0001$ \\
\hline Lactate dehydrogenase (LDH), $286.5 \mathrm{U} / \mathrm{L}$ and above & 3.122 & 1.7636 & 5.5248 & $<0.0001$ \\
\hline C-reactive protein (CRP), $0.0275 \mathrm{~g} / \mathrm{L}$ and above & 2.518 & 1.3706 & 4.6274 & 0.0029 \\
\hline Procalcitonin (PCT), $0.085 \mathrm{pg} / \mathrm{mL}$ and above & 3.347 & 1.8878 & 5.9347 & $<0.0001$ \\
\hline Troponin, $5.9 \mathrm{ng} / \mathrm{L}$ and above & 3.555 & 2.0318 & 6.2195 & $<0.0001$ \\
\hline
\end{tabular}

$\mathrm{Cl}$, Confidence Interval 

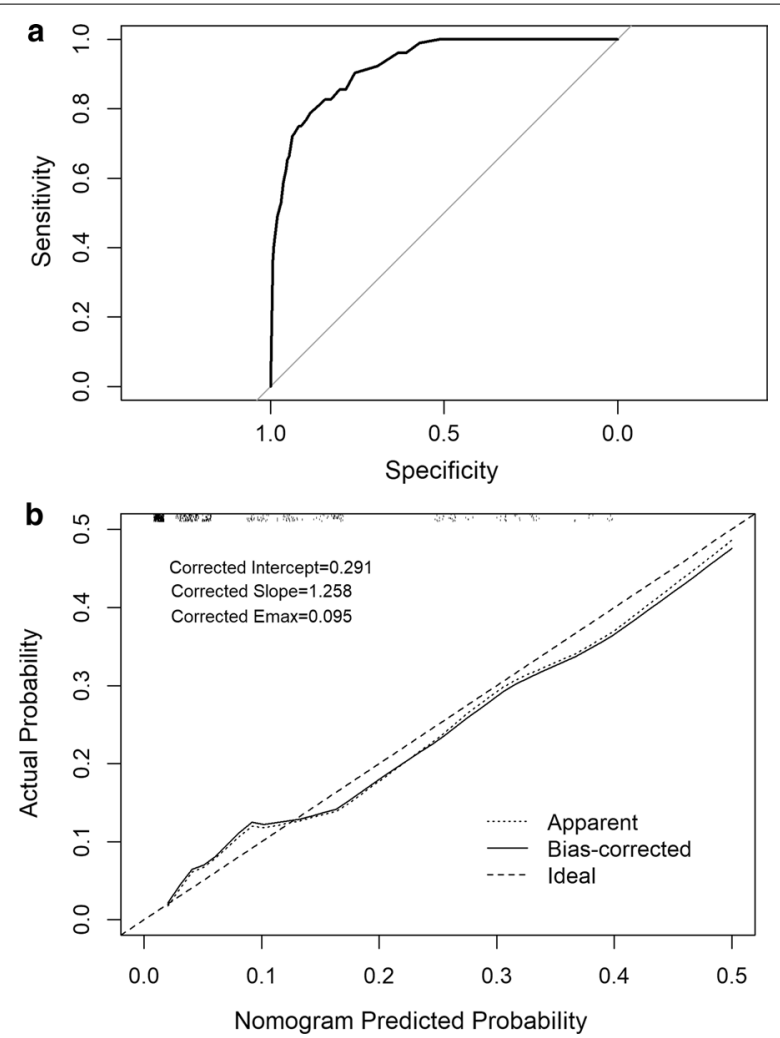

Fig. 3 Receiver-operating characteristics (ROC) (a) and calibration curves (b) analysis of the nomogram

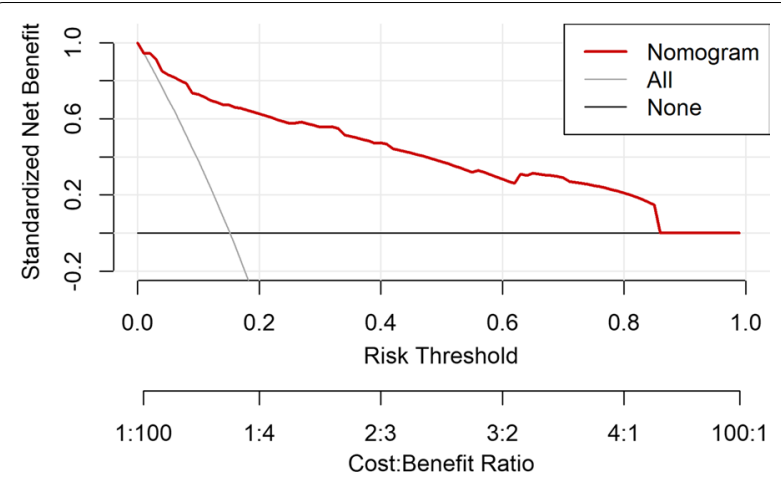

Fig. 4 Decision curve analysis of the nomogram predicting the risk of ICU need in hospitalized COVID-19 patients. The $x$-axis indicates the threshold probability, $y$-axis measures net benefit by adding true positive and subtracting false positive. A low-risk threshold probability might indicate that delaying the ICU admission is far more harmful than early admission; a higher threshold might indicate that waiting the parameters to reach critical levels is relatively more harmful than unnecessary ICU admission. When, threshold probability between $0.15-0.85$, predicting ICU admission by using our nomogram model would provide higher benefit than the admitting all the patients to the ICU (All i.e. treat all) or admitting none of the patients to the ICU (None i.e. treat none)

\section{Calibration}

Hosmer-Lemeshow goodness of fit test indicated that there is no significant difference between the predictive calibration curve and the ideal curve for predicting the ICU status of the patients (X-squared $=4.3284, \mathrm{df}=8$, $\mathrm{p}$-value $=0.8263)($ Fig. $3 \mathrm{~b})$. The calibration curves and Hosmer-Lemeshow test results indicates that the nomogram model is calibrated. In addition, significance of miscalibration of the model is evaluated using unreliability test $(\mathrm{p}=0.8197)$ and calibration test $(\mathrm{p}=0.1703)$ which indicates that the model is statistically well-calibrated.

\section{Discussion}

As it is known, COVID-19 has a mild-to-moderate course in most patients, but it progresses to severe illness in one-fifth of patients. Knowing which patients COVID19 will have a severe course is crucial in the management of patients and optimal use of medical resources such as hospital beds. Early identification of COVID-19 patients at high risk for serious disease development will enable patients to reach faster supportive care and treatment. On the other hand, determining patients with low risk of developing ICU need can assist physicians in the decision of discharge of these patients. Especially, the healthcare facilities with a low capacity of ICU beds have difficulties in the follow-up of patients. These facilities can prefer to transfer the patients with high risk for the development of ICU need to the further healthcare facilities with high capacity of ICU beds, while they can follow-up the patients with low risk for ICU need in their hospital wards. Therefore, we developed a simple and easy-to-use nomogram (and online calculator) that makes physician's decisions on the management of COVID-19 patients easier and provides ways of recognizing severe illness requiring ICU by using available and accessible values of patients on the first admission.

Several risk factors associated with the severity of disease have so far been reported in COVID-19 patients [13-17]. However, evaluating these risk factors by using a nomogram that predicts the severity of the disease can be more realistic and practical method for physicians. Nomograms are simple calculators, used commonly in the medicine, that could predict an individual numerical probability of a clinical event [9]. We constructed and validated a functional nomogram that incorporated five variables to predict the patients that carry a high risk for ICU admission by using baseline demographic, clinical, and laboratory parameters of the patients. The strongest nomogram was obtained with five independent variables consisting of SpO2, CRP, PCT, LDH, and troponin. The present nomogram has an excellent discriminative value with an AUC of 0.93 in the prediction of the individual risk of ICU admission in hospitalized patients 
with COVID-19. We demonstrated the optimal performance of the model by validation. As these five components are easily accessible parameters in the emergency department, this nomogram can help physicians to classify patients properly and decide on the proper follow up strategy.

In our study, the parameter with the greatest impact on ICU admission is SpO2 (100 points). As it is known, COVID-19 is a respiratory tract disease that can cause multisystem involvement and hypoxia is one of its most expected symptoms that also indicates the severity of the disease [1]. We demonstrated that the need of ICU admission is 4.4 (95\% CI 2.48-7.72) times higher in patients with a $\mathrm{SpO} 2$ of $94.5 \%$ and below compared to others. Saturation was reported as an independent risk factor for mortality in COVID-19 patients [18, 19]. Like our study, Acar et al. reported a 2.81-fold increase in mortality in patients having $\mathrm{SpO} 2$ between 89 and $94 \%$ and an 8.81-fold increase in $\mathrm{SpO} 2$ of $88 \%$ or less. Dyspnea and tachypnea, which are indicators of hypoxia, have also been reported to be associated with disease severity and unfavorable outcomes [20-22]. Hyperinflammation and its impact on severe COVID-19 was shown in COVID19 pandemic [23]. Both CRP and PCT are inflammatory parameters and reported to be associated with severe illness or mortality in patients with COVID-19 in many studies [16, 19, 21, 24-30]. In this study, CRP and PCT were detected as independent factors associated with increased ICU admission risk and both were included in the nomogram. Although not identical, our results contain similar findings with previous reports. In our study, the risk for ICU need is 2.5 (95\% CI 1.37-4.63) times higher in patients with a CRP of $0.0265 \mathrm{~g} / \mathrm{L}$ or more when compared to those having lower CRP. Previous studies detected different cut-off values and ranges for CRP as a predictor of disease severity [19, 21, 24-27].

In our model, LDH is another marker to predict ICU need. The patients with an LDH of $286.5 \mathrm{U} / \mathrm{L}$ and above had 3.1 (95\% CI 1.76-5.53) times higher risk for severe infection requiring ICU follow up. LDH is a tissue damage marker and is released from cells into the serum in the existence of cell damage. Therefore, LDH may help detecting tissue damage in the onset of COVID-19 infection. Similar to our study, some previous studies reported LDH as a predictive marker for severe illness [20, 24, 27, $31,32]$. Troponin I is also one of the predictors in our nomogram. There is growing evidence on the unfavorable impact of cardiac events associated with COVID19 on prognosis. Some studies reported that troponin I was detected significantly higher in those who died or needed mechanical ventilation compared to survivors or patients who did not need mechanical ventilation [33,
34]. Troponin I may be an early indication of worsening in COVID-19 patients without a detectable cardiac event.

In the literature, there are studies proposing nomograms and/or different models to predict serious illness or death in COVID-19 patients [19, 21, 22, 24, 25, 35-37]. Age was reported as a predictor for severe COVID-19 or mortality in previous studies and is included in most of the prediction models [16-21, 24, 25, 32, 35, 38, 39]. We found an increased risk of severe infection requiring ICU admission in the patients 56.5 years of age and older. However, it was not found significant enough to be included in the final nomogram as an optimal predictor for severe illness requiring ICU follow up. The nomogram developed by Gong et al. for early identification of cases with a high risk of progression to severe COVID19 included older age with six laboratory parameters [24]. Yu et al. developed a nomogram incorporated age and chest CT characteristics to define severe COVID19 in non-severe hospitalized COVID-19. Liang W et al. reported that they developed and validated a clinical risk score named as COVID-GRAM with ten parameters (chest radiographic abnormality, hemoptysis, dyspnea, age, unconsciousness, number of comorbidities, cancer histories, neutrophil-to-lymphocyte ratio, LDH, and direct bilirubin) [20]. However, like our study, there are also studies in which age was not determined as a predictor for disease severity [22, 26, 28, 40]

In addition to older age, the presence of comorbidities such as hypertension, cardiovascular disease and diabetes are risk factors for a severe disease requiring ICU in COVID-19 patients [16, 17, 31]. The association between comorbid diseases and the development of severe infection was indicated in previous studies $[14,16,17,31,41$, 42]. Some were severity and mortality risk scores [16, 19, $22,31,32,37]$. Although we found that comorbid diseases were more frequent in severe cases requiring ICU follow up than the patients without ICU need, comorbidities were not identified as optimal predictors during the development process of the nomogram.

There are many strengths of our study. It is demonstrated that COVID-19 is a multisystem disease with uncontrolled inflammatory response and tissue damage. Therefore, finding different parameters from different pathways; $\mathrm{SpO} 2$ representing the respiratory system, CRP and PCT as inflammatory markers, LDH as markers of tissue damage, and troponin from cardiac involvement, proves the good fitness of our model to this disease's pathogenesis. Multi-disciplinary nature of our model provides the opportunity to make an integrated decision related with the follow-up strategy for the patient. These five parameters can easily be obtained in emergency departments or outpatient clinics. The nomogram in our study exhibited an good discriminative 
power with an corrected C-index of 0.91 in the prediction of severe illness requiring ICU follow up on admission. Its performance is calibrated [10, 43, 44].

Our study has some limitations. Firstly, some patients are directly admitted to the ICU or immediately transferred to ICU on admission. To overcome this problem, we did not include patients who were directly hospitalized in the intensive care unit or those who were transferred to intensive care within the first $24 \mathrm{~h}$ of hospitalization. Secondly, we performed the study in a referral center. The more extensive, multicenter, and large sample sized studies will be better to represent the whole population. Thirdly, the study was designed retrospectively. Some cases had incomplete data. A large number of patients could not be included in the model development process and others had also missing data in acceptable limits.

\section{Conclusion}

We developed a nomogram for the prediction of severe illness requiring ICU with good distinctive power. The present nomogram supports the clinician through available clinical and laboratory parameters obtained at the first admission. The clinician can decide more easily where the patient should be followed, in the hospital or outside (at home, in the isolation institute or nursing home), or further healthcare facilities. The primary or secondary care facilities can use the present nomogram when they first examine the patients to decide whether the patients have a high risk for the development of ICU need or not, or whether they should transfer the patients or not. The patients who have severe illness or have a potential for worsening in the following days can be transferred earlier to the appropriate clinic. The patients with low risk for severe illness and ICU need can be more confidently discharged from hospital in facilities with low-bed capacity due to not expecting that patient's disease will not probably progress to severe illness. Finally, the optimal use of hospital beds can be provided by preventing unnecessarily long hospitalizations in those who were predicted low risk for the severe outcome, especially in countries with limited sources in terms of hospital beds or financial capacity.

\footnotetext{
Abbreviations

COVID-19: Coronavirus Disease-2019; SARS-CoV-2: Severe Acute Respiratory Syndrome Coronavirus-2; CT: Computer tomography; WHO: World Health Organization; ARDS: Acute respiratory distress syndrome; PCR: Polymerase chain reaction assay; ICU: Intensive care unit; SD: Standard deviation; IQR: Inter-quartile range; OR: Odds ratio; Cl: Confidence interval.
}

\section{Supplementary Information}

The online version contains supplementary material available at https://doi. org/10.1186/s12879-021-06656-w.

Additional file 1: Table S1. Score assignment for each variable included in nomogram and calculation the risk for ICU admission based on total point. Table S2. Calibration indexes of the nomogram model.

\section{Acknowledgements}

The authors would like to thank all staff of Ankara City Hospital who worked with all their strength during the pandemic.

\section{Authors' contributions}

All authors have made substantial contributions to the study. Study concept and design (BK and $R G$ ), acquisition of the data (IH, AA, AKK, HB, IA, EA, DE, $\mathrm{SI}, \mathrm{AA}, \mathrm{AK}, \mathrm{AAS}, \mathrm{MA}, \mathrm{SK}, \mathrm{IOT}, \mathrm{EA}, \mathrm{BO}, \mathrm{MNM}, \mathrm{YAB}, \mathrm{EMS}, \mathrm{DG}, \mathrm{BD}, \mathrm{SG}, \mathrm{EG}, \mathrm{RB}, \mathrm{OA}$, $D A, O I, T B)$, analysis and interpretation of the data (BK, RG and $I H)$, drafting of the manuscript (BK and $R G$ ), critical revision of the manuscript for important intellectual content (RG, FE), statistical expertise, and acquisition of funding (BK, RG). BK and RG revised the manuscript. All authors read and approved the final manuscript.

\section{Funding}

The authors declare that the current study has not been funded by any institution.

\section{Availability of data and materials}

The anonymized datasets generated and/or analyzed during the current study are not publicly available due to Turkey's Personal Data Protection Law No. 6698, but are available from the corresponding author upon reasonable request.

\section{Declarations}

Ethical approval and consent to participate

The research was conducted ethically in accordance with the World Medical Association Declaration of Helsinki. The study was approved by the Turkish Ministry of Health and Ankara City Hospital, Ethical Committee 1 (Reference Number E1/783/2020). Any administrative permission was not required to access the raw data used in our study. The patient's data were anonymized before using it.

\section{Consent for publication}

Non-applicable.

\section{Competing interests}

The authors declare that they have no competing interests.

\section{Author details}

'Department of Infectious Disease and Clinical Microbiology, Ankara Yildirim Beyazit University, Ankara City Hospital, Bilkent Street no:1, Ankara 06800, Turkey. ${ }^{2}$ Department of Infectious Disease and Clinical Microbiology, Ankara City Hospital, Ankara, Turkey. ${ }^{3}$ Department of Infectious Disease and Clinical Microbiology, University of Health Sciences, Ankara City Hospital, Ankara, Turkey. ${ }^{4}$ Department of Internal Medicine, Ankara City Hospital, Ankara, Turkey. ${ }^{5}$ Department of Anesthesiology and Reanimation, Ankara City Hospital, Ankara, Turkey. ${ }^{6}$ Department of Anesthesiology and Reanimation and Intensive Care Unit, Ankara Yildirim Beyazit University, Ankara City Hospital, Ankara, Turkey. ${ }^{7}$ Department of Pulmonary Diseases, Ankara Yildirim Beyazit University, Ankara City Hospital, Ankara, Turkey. ${ }^{8}$ Department of General Surgery, Ankara City Hospital, Ankara, Turkey. ${ }^{9}$ Department of Anesthesiology and Reanimation, University of Health Sciences, Ankara City Hospital, Ankara, Turkey.

${ }^{10}$ Department of Pulmonary Diseases, Ankara City Hospital, Ankara, Turkey.

${ }^{11}$ Department of Microbiology, Ankara City Hospital, Ankara, Turkey. 


\section{References}

1. World Health Organization. Clinical management of COVID-19. Interim guidance, 27 May 2020. https://www.who.int/publications-detail/clini cal-management-of-covid-19. Licence CC BY-NC-SA 3.0 IGO. Accessed 10 July 2020.

2. Wu Z, McGoogan JM. Characteristics of and important lessons from the Coronavirus Disease (COVID-19) outbreak in China: summary of a report of 72314 cases from the Chinese Center for Disease Control and Prevention. JAMA. 2020. https://doi.org/10.1001/jama.2020.2648.

3. World Health Organization. Coronavirus disease (COVID-19) technical guidance: laboratory testing for 2019-nCoV in humans. https://www.who. int/emergencies/diseases/novel-coronavirus-2019/technical-guidance/ laboratory-guidance. Accessed 08 July 2020.

4. Beaujean A. BaylorEdPsych: R Package for Baylor University Educational Psychology Quantitative Courses. R package (Version 0.5) [Computer Sofware] Waco, TX: Baylor University; 2012. https://CRAN.R-project.org/ package $=$ BaylorEdPsych.

5. Jamshidian M, Jalal S, Jansen C. MissMech: an R package for testing homoscedasticity, multivariable normality, and missing completely at random (MCAR). J Stat Softw. 2014:56(6):1-31.

6. van Buuren S, Groothuis-Oudshoorn K. Mice: multivariable imputation by chained equations in R. J Stat Softw. 2011;45(3):1-67.

7. Kowarik A, Templ M. Imputation with the R package VIM. J Stat Softw. 2016:74(7):1-16. https://doi.org/10.18637/jss.v074.i07.

8. Hosmer DW, Lemeshow JS, Sturdivant RX. Model Building Strategies for Logistic Regression. In Applied logistic regression. 3th edition. In: Cressie NA, editor. Hoboken: Wiley; 2013:89.152

9. Lawless J, Singhal K. Efficient screening of nonnormal regression models. Biometrics. 1978;34(2):318-27. https://doi.org/10.2307/2530022.

10. Harrell FE. rms: Regression Modeling Strategies. R package version 6.2-0; 2021. https://CRAN.R-project.org/package $=$ rms.

11. Robin X, Turck N, Hainard A, Tiberti N, Lisacek F, Sanchez JC, Müller M. pROC: an open-source package for R and S+ to analyze and compare ROC curves. BMC Bioinform. 2011;12:77. https://doi.org/10.1186/ 1471-2105-12-77.

12. Moons KG, Altman DG, Reitsma JB, loannidis JP, Macaskill P, Steyerberg EW, Vickers AJ, Ransohoff DF, Collins GS. Transparent Reporting of a multivariable prediction model for Individual Prognosis or Diagnosis (TRIPOD): explanation and elaboration. Ann Intern Med. 2015;162(1):W1-73. https:// doi.org/10.7326/M14-0698.

13. Levy TJ, Richardson S, Coppa K, Barnaby DP, McGinn T, Becker LB, et al. Development and validation of a survival calculator for hospitalized patients with COVID-19. Preprint. medRxiv. 2020;2020.04.22.20075416.

14. Du R-H, Liang L-R, Yang C-Q, Wang W, Cao T-Z, Li M, et al. Predictors of mortality for patients with COVID-19 pneumonia caused by SARS-CoV-2: a prospective cohort study. Eur Respir J. 2020;55(5):2000524.

15. Mizumoto K, Chowell G. Estimating risk for death from Coronavirus disease, China, January-February 2020. Emerg Infect Dis. 2020;26(6):1251-6.

16. Wang K, Zuo P, Liu Y, Zhang M, Zhao X, Xie S, et al. Clinical and laboratory predictors of in-hospital mortality in patients with COVID-19: a cohort study in Wuhan, China. Clin Infect Dis. 2020; ciaa538.

17. Ruan Q, Yang K, Wang W, Jiang L, Song J. Clinical predictors of mortality due to COVID-19 based on an analysis of data of 150 patients from Wuhan, China. Intensive Care Med. 2020;46(5):846-8.

18. Yadaw AS, Li YC, Bose S, lyengar R, Bunyavanich S, Pandey G. Clinical predictors of COVID-19 mortality. Preprint. medRxiv. 2020; 2020.05.19.20103036.

19. Acar HC, Can G, Karaali R, Börekçi Ş, Balkan Ii, Gemicioğlu B, Konukoğlu D, Erginöz E, Erdoğan MS, Tabak F. An easy-to-use nomogram for predicting in-hospital mortality risk in COVID-19: a retrospective cohort study in a university hospital. BMC Infect Dis. 2021;21(1):148.

20. Liang W, Liang H, Ou L, Chen B, Chen A, Li C, Li Y, Guan W, Sang L, Lu J, Xu Y, Chen G, Guo H, Guo J, Chen Z, Zhao Y, Li S, Zhang N, Zhong N, He J. China Medical Treatment Expert Group for COVID-19. Development and validation of a clinical risk score to predict the occurrence of critical illness in hospitalized patients with COVID-19. AMA Intern Med. 2020;180(8):1081-9. https://doi.org/10.1001/jamainternmed.2020.2033.

21. Nguyen $Y$, Corre F, Honsel V, Curac S, Zarrouk V, Burtz CP, Weiss E, Moyer JD, Gauss T, Grégory J, Bert F, Trichet C, Peoc'h K, Vilgrain V, Rebours V, Fantin B, Galy A. A nomogram to predict the risk of unfavourable outcome in COVID-19: a retrospective cohort of 279 hospitalized patients in Paris area. Ann Med. 2020;52(7):367-75. https://doi.org/10.1080/07853890. 2020.1803499 (Epub 2020 Aug 14).

22. Zhou Y, He Y, Yang H, Yu H, Wang T, Chen Z, Yao R, Liang Z. Exploiting an early warning Nomogram for predicting the risk of ICU admission in patients with COVID-19: a multi-center study in China. Scand J Trauma Resusc Emerg Med. 2020;28(1):106.

23. Qin C, Zhou L, Hu Z, Zhang S, Yang S, Tao Y, Xie C, Ma K, Shang K, Wang W, Tian DS. Dysregulation of immune response in patients with coronavirus 2019 (COVID-19) in Wuhan, China. Clin Infect Dis. 2020;71(15):762-8. https://doi.org/10.1093/cid/ciaa248.

24. Gong J, Ou J, Qiu X, Jie Y, Chen Y, Yuan L, Cao J, Tan M, Xu W, Zheng F, Shi Y, Hu B. A tool for early prediction of severe coronavirus disease 2019 (COVID-19): a multicenter study using the risk nomogram in Wuhan and Guangdong, China. Clin Infect Dis. 2020;71(15):833-40.

25. Weng Z, Chen Q, Li S, Li H, Zhang Q, Lu S, Wu L, Xiong L, Mi B, Liu D, Lu $M$, Yang $D$, Jiang $H$, Zheng S, Zheng $X$. ANDC: an early warning score to predict mortality risk for patients with Coronavirus Disease 2019. J Transl Med. 2020;18(1):328.

26. Chen H, Chen R, Yang H, Wang J, Hou Y, Hu W, Yu J, Li H. Development and validation of a nomogram using on admission routine laboratory parameters to predict in-hospital survival of patients with COVID-19. J Med Virol. 2021;93(4):2332-9. https://doi.org/10.1002/jmv.26713 (Epub 2020 Dec 23).

27. Wu G, Yang P, Xie Y, Woodruff HC, Rao X, Guiot J, Frix AN, Louis R, Moutschen M, Li J, Li J, Yan C, Du D, Zhao S, Ding Y, Liu B, Sun W, Albarello F, D'Abramo A, Schininà V, Nicastri E, Occhipinti M, Barisione G, Barisione E, Halilaj I, Lovinfosse P, Wang X, Wu J, Lambin P. Development of a clinical decision support system for severity risk prediction and triage of COVID-19 patients at hospital admission: an international multicentre study. Eur Respir J. 2020;56(2):2001104. https://doi.org/10.1183/13993003. 01104-2020.

28. Pan D, Cheng D, Cao Y, Hu C, Zou F, Yu W, Xu T. A predicting nomogram for mortality in patients with COVID-19. Front Public Health. 2020;8:461. https://doi.org/10.3389/fpubh.2020.00461.

29. Pan F, Yang L, Li Y, Liang B, Li L, Ye T, Li L, Liu D, Gui S, Hu Y, Zheng C. Factors associated with death outcome in patients with severe coronavirus disease-19 (COVID-19): a case-control study. Int J Med Sci. 2020;17(9):1281-92. https://doi.org/10.7150/ijms.46614.

30. Xu JB, Xu C, Zhang RB, Wu M, Pan CK, Li XJ, Wang Q, Zeng FF, Zhu S. Associations of procalcitonin, C-reaction protein and neutrophil-to-lymphocyte ratio with mortality in hospitalized COVID-19 patients in China. Sci Rep. 2020;10(1):15058. https://doi.org/10.1038/s41598-020-72164-7.

31. Ji D, Zhang D, Xu J, Chen Z, Yang T, Zhao P, Chen G, Cheng G, Wang Y, Bi J, Tan L, Lau G, Qin E. Prediction for progression risk in patients with COVID19 pneumonia: the CALL score. Clin Infect Dis. 2020;71(6):1393-9. https:// doi.org/10.1093/cid/ciaa414.

32. Li L, Fang $X$, Cheng L, Wang $P$, Li S, Yu H, Zhang Y, Jiang N, Zeng T, Hou C, Zhou J, Li S, Pan Y, Li Y, Nie L, Li Y, Sun Q, Jia H, Li M, Cao G, Ma X. Development and validation of a prognostic nomogram for predicting in-hospital mortality of COVID-19: a multicenter retrospective cohort study of 4086 cases in China. Aging (Albany NY). 2021;13(3):3176-89.

33. Zhou F, Yu T, Du R, Fan G, Liu Y, Liu Z, et al. Clinical course and risk factors for mortality of adult inpatients with COVID-19 in Wuhan, China: a retrospective cohort study (published correction appears in Lancet. 2020 Mar 28;395(10229):1038) (published correction appears in Lancet. 2020 Mar 28;395(10229):1038). Lancet. 2020;395(10229):1054-1062.

34. Kermali M, Khalsa RK, Pillai K, Ismail Z, Harky A. The role of biomarkers in diagnosis of COVID-19_a systematic review. Life Sci. 2020;254: 117788. https://doi.org/10.1016/j.lfs.2020.117788.

35. Yu Y, Wang X, Li M, Gu L, Xie Z, Gu W, Xu F, Bao Y, Liu R, Hu S, Hu M, Hu C. Nomogram to identify severe coronavirus disease 2019 (COVID-19) based on initial clinical and CT characteristics: a multi-center study. BMC Med Imag. 2020;20(1):111.

36. Dong YM, Sun J, Li YX, Chen Q, Liu QQ, Sun Z, Pang R, Chen F, Xu BY, Manyande A, Clark TG, Li JP, Orhan IE, Tian YK, Wang T, Wu W, Ye DW. Development and validation of a nomogram for assessing survival in patients with COVID-19 pneumonia. Clin Infect Dis. 2021;72(4):652-60. https://doi. org/10.1093/cid/ciaa963.

37. Dai Z, Zeng D, Cui D, Wang D, Feng Y, Shi Y, Zhao L, Xu J, Guo W, Yang Y, Zhao X, Li D, Zheng Y, Wang A, Wu M, Song S, Lu H. Prediction of 
COVID-19 patients at high risk of progression to severe disease. Front Public Health. 2020;8:574915.

38. Wang D, Hu B, Hu C, Zhu F, Liu X, Zhang J, et al. Clinical characteristics of 138 hospitalized patients with 2019 novel coronavirus-infected pneumonia in Wuhan, China. Jama. 2020;323(11):1061-9.

39. Wang B, Li R, Lu Z, Huang Y. Does comorbidity increase the risk of patients with COVID-19: evidence from meta-analysis. Aging (Albany NY). 2020;12(7):6049-57.

40. Zhou Y, He Y, Yang H, Yu H, Wang T, Chen Z, et al. Development and validation a nomogram for predicting the risk of severe COVID-19: A multicenter study in Sichuan, China. PLoS One. 2020;15(5):233328.

41. Hu Y, Sun J, Dai Z, Deng H, Li X, Huang Q, et al. Prevalence and severity of corona virus disease 2019 (COVID-19): a systematic review and metaanalysis. J Clin Virol. 2020;127:104371.
42. Shi Y, Yu X, Zhao H, Wang H, Zhao R, Sheng J. Host susceptibility to severe COVID-19 and establishment of a host risk score: findings of 487 cases outside Wuhan. Crit Care. 2020;24(1):108.

43. Hosmer DW, Hjort NL. Goodness-of-fit processes for logistic regression: simulation results. Stat Med. 2002;21:2723-38.

44. Nattino G, Finazzi S, Bertolini G, Rossi C, Carrara G. givitiR: The GiViTI Calibration Test and Belt. R package version 1.3. [Computer Sofware] 2017. https://CRAN.R-project.org/package=givitiR.

\section{Publisher's Note}

Springer Nature remains neutral with regard to jurisdictional claims in published maps and institutional affiliations.
Ready to submit your research? Choose BMC and benefit from:

- fast, convenient online submission

- thorough peer review by experienced researchers in your field

- rapid publication on acceptance

- support for research data, including large and complex data types

- gold Open Access which fosters wider collaboration and increased citations

- maximum visibility for your research: over $100 \mathrm{M}$ website views per year

At BMC, research is always in progress.

Learn more biomedcentral.com/submissions 